\title{
Measuring rank robustness in scored protein interaction networks
}

\author{
Lyuba V. Bozhilova ${ }^{1}$, Alan V. Whitmore ${ }^{2}$, Jonny Wray ${ }^{2}$, Gesine Reinert ${ }^{1}$ and Charlotte M. Deane ${ }^{1^{*}}$ (D)
}

\begin{abstract}
Background: Protein interaction databases often provide confidence scores for each recorded interaction based on the available experimental evidence. Protein interaction networks (PINs) are then built by thresholding on these scores, so that only interactions of sufficiently high quality are included. These networks are used to identify biologically relevant motifs or nodes using metrics such as degree or betweenness centrality. This type of analysis can be sensitive to the choice of threshold. If a node metric is to be useful for extracting biological signal, it should induce similar node rankings across PINs obtained at different reasonable confidence score thresholds.

Results: We propose three measures—rank continuity, identifiability, and instability —-to evaluate how robust a node metric is to changes in the score threshold. We apply our measures to twenty-five metrics and identify four as the most robust: the number of edges in the step-1 ego network, as well as the leave-one-out differences in average redundancy, average number of edges in the step-1 ego network, and natural connectivity. Our measures show good agreement across PINs from different species and data sources. Analysis of synthetically generated scored networks shows that robustness results are context-specific, and depend both on network topology and on how scores are placed across network edges.

Conclusion: Due to the uncertainty associated with protein interaction detection, and therefore network structure, for PIN analysis to be reproducible, it should yield similar results across different confidence score thresholds. We demonstrate that while certain node metrics are robust with respect to threshold choice, this is not always the case. Promisingly, our results suggest that there are some metrics that are robust across networks constructed from different databases, and different scoring procedures.
\end{abstract}

Keywords: Confidence scores, Protein-protein interactions, Protein interaction networks, Ranking, Robustness

\section{Background}

Protein interaction networks (PINs) are models of cellular architecture in which proteins are represented by nodes and the biologically meaningful interactions between them are represented by edges. PIN analysis has a wide range of applications in bioinformatics [1-3], and in particular in drug discovery [4-6]. For example, it can be used to predict protein function [7-10] and disease relevance [11-13], as well as to identify possible drug targets, especially in the case of multi-target drug discovery [14]. A common aim of PIN analysis is the identification of key

*Correspondence: deane@stats.ox.ac.uk

'Department of Statistics, University of Oxford, 24-29 St Giles', OX1 3LB Oxford, UK

Full list of author information is available at the end of the article actors in the network, e.g. for the purposes of drug target choice [15-17].

PINs can be built using both physical and/or functional interactions, which can in turn be obtained from a range of experimental and in silico techniques $[18,19]$. There exist numerous databases of protein interactions which vary in data type, data collection methods, and content curation [20,21]. High-throughput interaction data based on yeast two-hybrid assays [22], tandem affinity purification [23] or gene co-expression [24] are often subject to high false positive and false negative rates (e.g. [25-27]). Different approaches to data curation are used to correct for this. Some databases, such as STRING [28], HitPredict [29], IntAct [30], and HIPPIE [31], quantify the strength of the supporting evidence for each reported interaction by assigning a confidence score to it. These confidence scores are often a combination (c) The Author(s). 2019 Open Access This article is distributed under the terms of the Creative Commons Attribution 4.0 International License (http://creativecommons.org/licenses/by/4.0/), which permits unrestricted use, distribution, and reproduction in any medium, provided you give appropriate credit to the original author(s) and the source, provide a link to the Creative Commons license, and indicate if changes were made. The Creative Commons Public Domain Dedication waiver (http://creativecommons.org/publicdomain/zero/1.0/) applies to the data made available in this article, unless otherwise stated. 
of different sub-scores (e.g. based on different evidence channels in STRING), each of which is calculated in a custom, source-specific way. The final combined scores are usually scaled between 0 and 1 but are not readily interpretable, and, as we illustrate, tend not to be comparable across databases. Such scores are designed to provide a comparison between different interactions (an interaction with confidence score of 0.40 is supported by weaker evidence than an interaction with score of $0.90)$, so researchers can control strength of evidence by imposing a score threshold. STRING, for example, suggests thresholds of 0.15 (low confidence), 0.40 (medium confidence), 0.70 (high confidence) or 0.90 (highest confidence), whereas HitPredict identifies all interactions scoring below 0.28 as medium-high confidence, and interactions scoring above as high confidence.

Due to the wide range of available resources and quality assessment tools for protein interaction data a number of different networks can be built to model the same part of the interactome. Even when the data type and source are fixed, a threshold on data quality is often chosen, either by database curators, or explicitly by researchers during network construction. Only interactions which meet that threshold contribute to the final network structure (see [28] for a discussion on scores and thresholding, and [32] for a particular example). Such data preprocessing choices play an important role in PIN structure and we show that they can have an effect on any further network analysis.

Given interaction detection error rates, and the incomplete coverage of interaction detection experiments $[33,34]$, it is extremely unlikely that any one PIN is a perfect representation of the underlying biological processes it aims to model, regardless of how it is constructed. The effect of error, or noise, on networks, such as missing or misplaced edges, is an established research topic in network science [35], and is often studied via network perturbation [36]. In the context of PINs, stochastic models of noise on network edges have been used to re-score interactions [9], determine optimal score thresholds [37], and have been incorporated into community detection $[38,39]$. However, these models often rely on assumptions about the behaviour of the error: for example, they may assume that interactions are equally likely to be detected regardless of the properties of the proteins involved. Such assumptions do not necessarily hold in the context of protein interaction detection [40]. Moreover, any approach which incorporates interaction scores directly within PINs (e.g. by modelling them as weighted networks), implicitly relies both on the interpretability and the accuracy of the scores themselves, both of which can change over time and across data sources. The relative scarcity of high quality interaction data and lack of sufficiently good PIN-like random network models further complicate the validation of such approaches.
Rather than considering a single network, the observation of which is subject to a difficult to model noise process, assessing the robustness of a PIN analysis pipeline can be done by repeating the analysis across different networks representing the same part of the interactome. One way to do this would be to consider building different networks from the same scored interaction database by varying the confidence score threshold. We postulate that network features which are persistent across different thresholds are more likely to be informative of the biological state of interest than features which are only present at isolated thresholds. This hypothesis is in line with network research in neuroscience, where a similar heuristic is employed to identify which parts of a brain network are active across different observations [41].

In this paper we provide a framework for assessing the robustness of node metrics to threshold choice. Our framework is based on a measure of rank similarity described by [42]. We introduce three robustness measures-rank continuity, identifiability, and instability - which can be used to quantify how consistent a node metric is across different thresholds. Our methodology is particularly relevant to cases where a node metric is used to identify highly ranking nodes, which may correspond to key proteins in a particular process, for example for the purposes of drug target identification [16].

By analysing the effects of threshold change on a set of twenty-five node metrics across four scored PINs we show that some node metrics tend to be more robust-and are therefore possibly more relevant to biological researchthan others. The node metrics studied include standard node centralities, such as degree and betweenness, as well as leave-one-out difference (LOUD) metrics, which measure the effect of isolating a node on global network summaries such as the global clustering coefficient. Some of the metrics are based on ego networks, i.e. networks formed by taking a node and its nearby neighbours (see Methods for details). We identify the number of edges in the step-one ego network, and LOUD average redundancy [43], LOUD number of edges in the step-one ego network, and LOUD natural connectivity [44], as significantly more robust to threshold choice than more commonly used metrics, such as local clustering coefficient, betweenness centrality, and in some cases even degree.

Promisingly, our results show good agreement between networks from different organisms and databases. Complemented with analysis of synthetic data, we further show that robustness depends both on network topology and on score allocation across network edges.

\section{Results}

\section{Thresholding effects}

The reliability of a detected interaction between two proteins is often quantified by a confidence score, with 
lower scores corresponding to weaker interaction evidence. When PINs are constructed from such data, a threshold on the confidence scores is usually applied in an attempt to filter out spurious interactions. While it is possible to incorporate the confidence scores as edge weights in the network, these scores represent neither interaction strength, nor distance, making classical weighted network analysis techniques difficult to interpret. Moreover, confidence scores vary across databases, both in their derivation and interpretation, as well as in their values.

Confidence scores are designed specifically to allow researchers a degree of control over data quality, usually through thresholding. Threshold choice over the confidence scores introduces a trade-off between the numbers of false positive and false negative interactions. A low threshold may introduce many interactions which have been detected experimentally, but which have no biological relevance, while a high one will reduce the number of such false positives, but may also lead to more genuine interactions being excluded from the network.

Imposing different thresholds will affect PIN structure, and may affect PIN analysis in complex, and potentially difficult to predict, ways. Some metrics, such as edge density, node degree, and natural connectivity, will decrease monotonically with threshold increase. Other network metrics, such as clustering coefficients and betweenness centrality, do not necessarily behave monotonically and it is unclear how to predict their rate of change (or even its direction) between thresholds.
To examine the effect of threshold choice we considered three full organism networks obtained from the STRING database-Plasmodium vivax (PVX), Escherichia coli (ECOLI), and Saccharomyces cerevisiae (YEAST). STRING suggests using one of four thresholds as a default-low (0.15), medium (0.40), high (0.70), and highest (0.90). For each threshold, an unweighted network between the proteins is constructed which includes only those edges for which the score is at least as high as the threshold. The average degree in each of the three STRING networks analysed decreased with increasing thresholds, from over 200 at low confidence, to under 25 at highest confidence (Fig. 1a). In the PVX network, the average local clustering decreased monotonically from 0.50 down to 0.20 across the four suggested thresholds. However, in the ECOLI network, average local clustering increased from 0.24 (low confidence) to 0.40 (high confidence), before decreasing down to 0.35 (highest confidence). In the YEAST network, the average local clustering remained stable around 0.27 between low and medium confidence, and then steadily increased to 0.36 at the highest confidence threshold before dropping off again (Fig. 1b). Unlike average degree, average local clustering is non-linear, and is heavily influenced by lowdegree nodes in sparse networks. A small number of edge deletions can dramatically change the local clustering coefficients of such nodes, making it difficult to predict a priori how the average local clustering will change with the threshold.



\section{B}

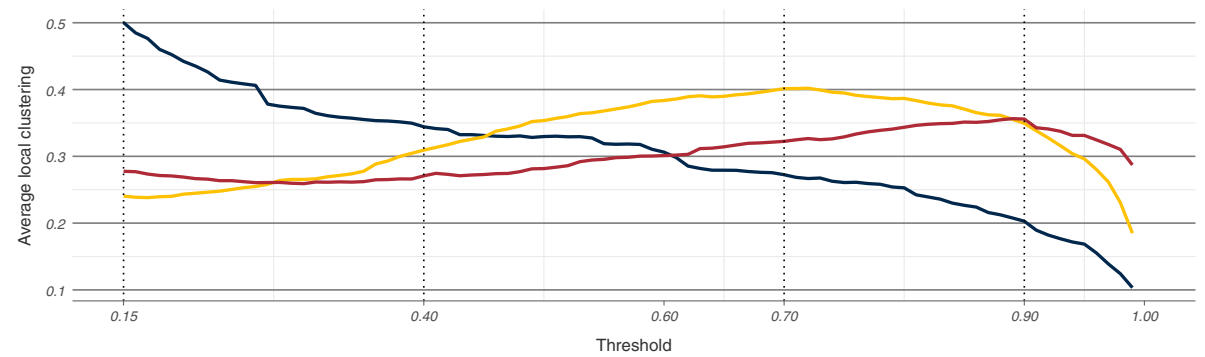

Fig. 1 Thresholding effects in STRING networks. Average degree (a) and average local clustering coefficient (b) as functions of the threshold in the three STRING networks. The dotted vertical lines correspond to the four default STRING threshold values 
Figure 1 illustrates two aspects of scored PINs: firstly that node metrics can vary significantly in their raw values across thresholds, and secondly, that this variation is qualitatively different for different metrics. The incomplete coverage and experimental error of interaction detection techniques imply that it is most unlikely that any particular thresholded network describes perfectly all biologically relevant interactions and only them. Therefore, any robust, biologically informative PIN analysis pipeline should ideally show agreement in results obtained across at least a narrow range of different thresholds. In the context of using node metrics to identify key proteins in a network, such an agreement may translate to identifying the same set of highest ranking nodes.

We analysed 25 node metrics, of which 12 were node centralities, and 13 were global network summaries which we redefined as leave-one-out difference (LOUD) metrics. Four scored PINs were considered, spanning three organisms and two databases-the three PINs from STRING, illustrated in Fig. 1 and the S. cerevisiae network obtained from HitPredict (HPRED). For each PIN, these metrics were calculated for all nodes in a set of 85 thresholded networks, obtained at equidistant thresholds from 0.15 (the lowest recorded) to 0.99 at 0.01 intervals. In addition we considered two synthetic scored networks-SYN-GNP based on a Bernoulli random graph, and SYN-PVX, based on a re-scored subset of the PVX network. The node rankings induced by a metric at each of the thresholded networks were used to assess the metric's rank robustness.

\section{Rank continuity}

PIN analysis often aims to identify key proteins in a particular biological process or context, for example by studying which nodes in the network attain high values across different node metrics. This problem relates to the node rankings induced by the metrics ("Which are the nodes of highest degree?"), rather than to exact metric values ("What is the degree of these nodes?").

Exact metric values can be difficult to interpret and will vary both between PINs and with the PIN confidence score threshold. For example, ubiquitin (YLL039C) has degree between 262 and 4254 across different thresholds of the YEAST network (values obtained at score thresholds 0.99 and 0.15 respectively). These values are vastly different, and not easily interpretable or comparable outside the context of the particular thresholded networks they are obtained from. In contrast, the fact that ubiquitin is the single highest degree node across all thresholds for the scored YEAST network demonstrates its biological role more clearly.

We propose that for a node metric to be reliably indicative of the biological state described by a scored PIN, it should identify similar sets of key, i.e. highest ranking, proteins at a range of thresholds. In particular, rankings obtained at consecutive thresholds (e.g. at 0.40 , the proposed medium confidence threshold in STRING, and at the slightly higher 0.41 ) should be in good agreement. Large differences could imply that (a) the metric is too influenced by pre-processing decisions to be informative, or (b) that the confidence score distribution is highly concentrated between these two thresholds and moving from one to the other significantly changes network topology.

For each analysed node metric, rank similarity was measured using Trajanovski's $k$-similarity (see Methods) between each two consecutive thresholds, across all scored networks-the three STRING networks, the $S$. cerevisiae network from HitPredict, and two synthetic networks (Fig. 2, Additional file 1: S1-S4). In the analysed PINs, three different modes of behaviour were observed:

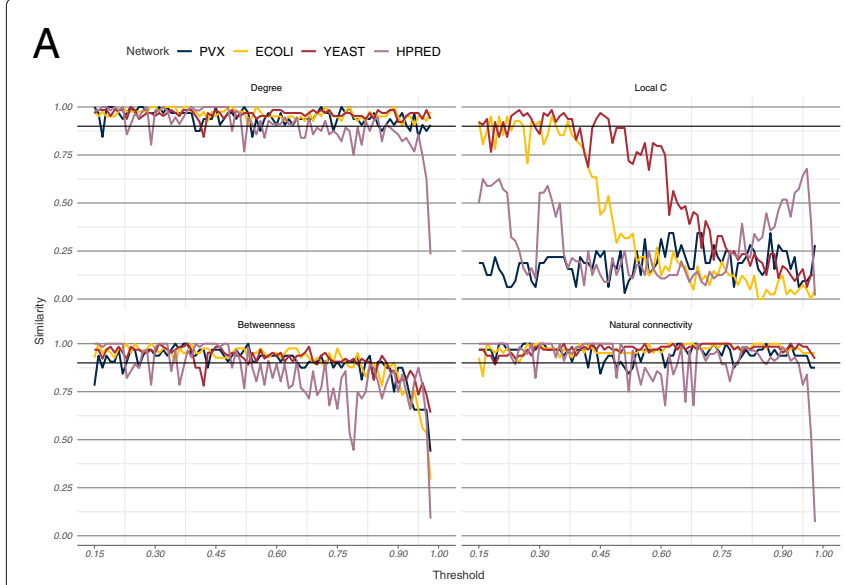

B

Network - SYN-GNP - SYN-PVX

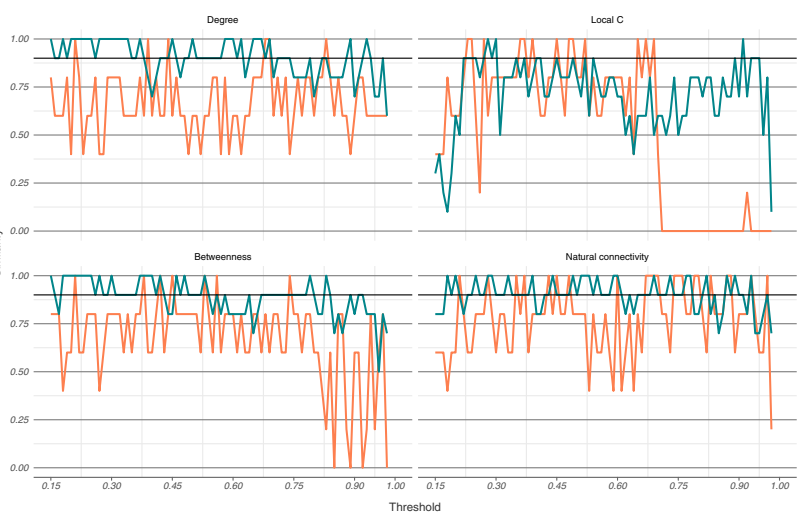

Fig. 2 Metric rank similarity between consecutive thresholds. a In the four PINs, metrics were either consistently stable (e.g. degree and LOUD natural connectivity), consistently unstable (e.g. local clustering coefficient), or showed decreasing stability (e.g. betweenness). b The synthetic network based on a randomly rescored subset of the PVX network, SYN-PVX, and the network based on a Bernoulli random graph, SYN-GNP, exhibited different behaviour, with metrics showing the least similarity across thresholds in the SYN-GNP network 
some metrics exhibited consistently high similarity, some consistently low, and for others $k$-similarity steadily decreased with threshold increase (Fig. 2a, Additional file 1: S1 and S2).

We propose a rank continuity measure based on how often $k$-similarity between consecutive thresholds reaches 0.90 (details in Methods). Continuity was measured for our set of twenty-five metrics (Methods and SI for details) across the six scored networks (Additional file 1: Tables S1 and S5). Between 7 and 16 metrics were found to have continuity measures over 0.90 for the medium-high confidence region in each of the scored PINs. The value of 0.90 was chosen since we believe robust metrics should produce nearly identical sets of high-ranking nodes across most pairs of consecutive thresholds. Seven metrics were found to have continuity measures over 0.90 in all four networks, and an additional four metrics had high continuity in three out of the four networks (all but the PVX network). Eleven of the twenty-five metrics-degree, redundancy, PageRank, harmonic closeness, LOUD natural connectivity, LOUD global clustering, LOUD average redundancy, and four of the ego-network based metricshad an average score across the four PINs above 0.90. Nine metrics, including the commonly used local clustering coefficient and betweenness centrality, did not achieve a high continuity measure in any of the four PINs.

Spearman rank correlations of the continuity measures (Additional file 1: Table S2) showed extremely good agreement between the three STRING networks (all correlations were above 0.95 ), and very good agreement between the STRING networks and the HPRED network (correlations between 0.89 and 0.92). These were higher than correlations between the STRING networks and either of the synthetic networks (between 0.30 and 0.68 ), suggesting that how edge scores are placed over the network (biased as opposed to random) plays an important role in metric rank continuity. Finally, continuity in the synthetic Bernoulli network, SYN-GNP, was considerably lower across all metrics (Fig. 2b, Additional file 1: Table S5). This implies that the metric continuity is sensitive to network structure-the reported continuity values will not necessarily hold for other types of networks (e.g. social, transport, etc.), where other node metrics may be more stable.

Our continuity analysis suggests that nearly half of the tested node metrics are robust to small threshold perturbation in PINs. However, incremental changes in the set of overlapping nodes between consecutive thresholds may result in a high continuity measure but low similarity between rankings at more distant thresholds. This can be undesirable, since often confidence scores are not readily interpretable and there may exist a wide range of permissible thresholds (e.g. anywhere between 0.15 and 0.90 in STRING).

\section{Rank identifiability}

In order to assess the robustness of a node metric across a range of medium-high confidence score thresholds, overall ranks were calculated and compared to ranks induced at single thresholds. These ranks were designed to represent the relative position (i.e. importance) of each node across a range of medium-high confidence thresholds, and were calculated as rank averages across the threshold region. For example, consider two proteins $\mathrm{X}$ and $\mathrm{Y}$. Suppose that for thresholds 0.60 to $0.70, \mathrm{X}$ is the highest degree node in the network, and $\mathrm{Y}$ is the second highest, and vice versa for thresholds 0.71 to 0.90 . Since it is more often the case that $\mathrm{Y}$ has higher degree than $\mathrm{X}, \mathrm{Y}$ would be the overall highest ranking node with respect to degree. We define a rank identifiability measure which quantifies the ability to recover the set of overall highest ranking nodes by considering any single threshold in the region. Our identifiability measure is based on an asymmetric version of $k$-similarity, which we introduce and call $\alpha$-relaxed $k$-similarity (defined in Methods). Intuitively, a rank identifiability measure of 0.90 implies that at least 90 of the 100 overall highest ranking nodes are also among the top 150 at any given threshold. So if only a single threshold was considered, it would still contain the majority of nodes which rank highly across the entire region.

In each of the three STRING networks, 5 (for PVX) or 6 (for YEAST and ECOLI) node metrics were found to have rank identifiability measures above 0.90 (Additional file 1: Table S6). In the HPRED network, where the medium-high confidence region is shorter than in STRING, identifiability measures were higher and 16 metrics attained a score above 0.90 . Of these, four metrics-redundancy, number of edges in the step-one ego network, LOUD natural connectivity, and LOUD average number of edges in the step-one ego network - had identifiability measures above 0.90 across all four PINs.

The similarities of rankings induced at thresholds outside the medium-high confidence region to the overall ranks were also calculated, although these similarities did not contribute to the rank identifiability scores. Since the overall ranks were calculated over the medium-high confidence region for each network, it is natural to expect $\alpha$-relaxed $k$-similarities to be higher within the region than outside it (Fig. 3, Additional file 1: S5-S8). This trend is observed even in the case of the truly randomised SYN-GNP network (Fig. 3b). In the HPRED network, for example, some metrics showed high rank similarity for thresholds as high as 0.45. This indicates that the exact boundaries of the region do not necessarily heavily influence identifiability results.

Like rank continuity, rank identifiability is a contextdependent property of network metrics. The three 


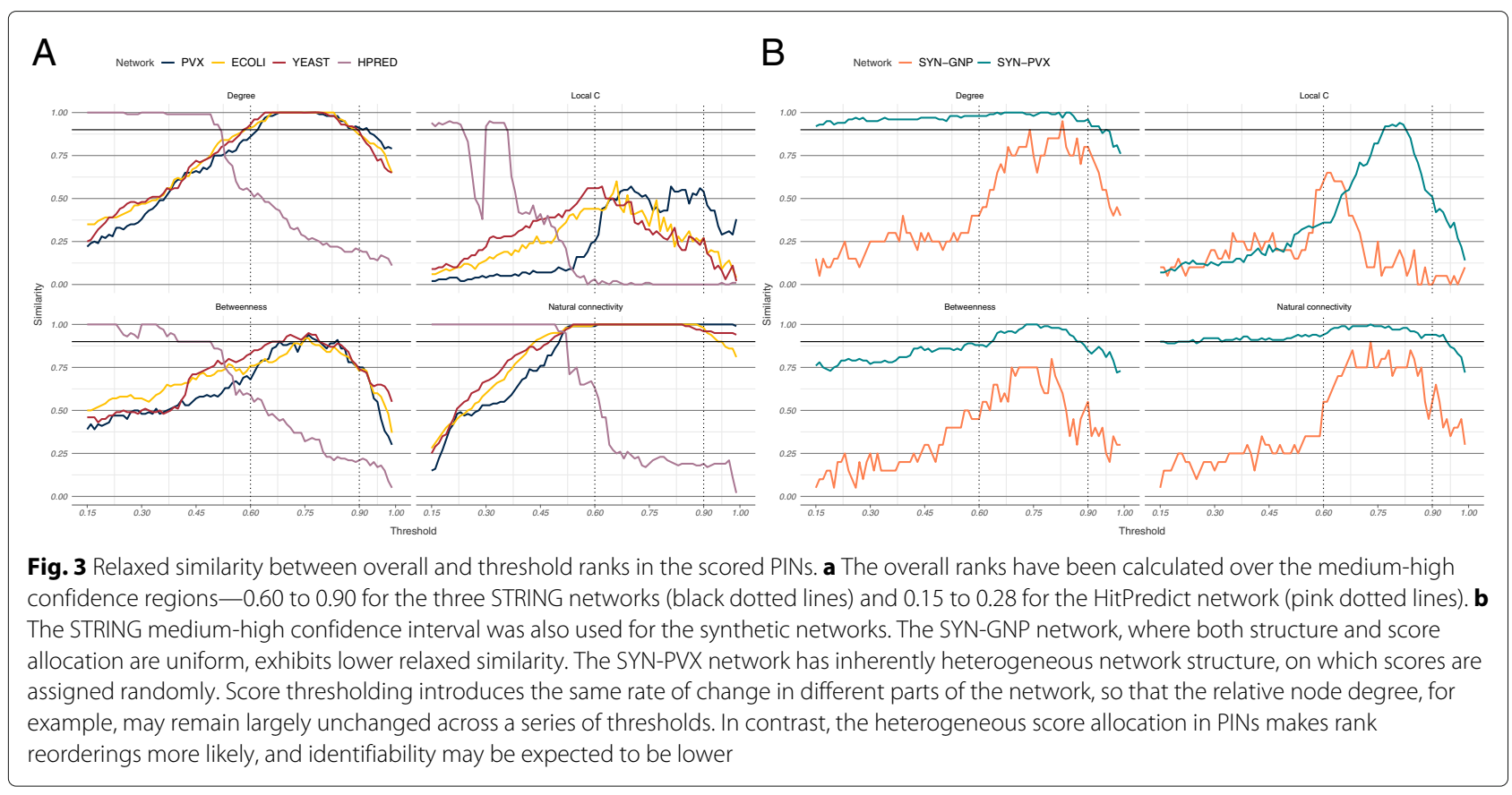

STRING networks closely agreed in identifiability measures (Spearman correlations between metric identifiability scores were all 0.94 and above), and were more similar to measures obtained from analysing the HPRED network (correlations above 0.81 ) than any of the synthetic networks (Additional file 1: Table S3).

While rank identifiability can be used to quantify rank conservation across many thresholds, it does not account for all types of rank variability. Intuitively, a metric which preserves the exact same ranking in the set of top $n$ nodes at every threshold is more robust than one in which the top node set is preserved, but re-ranked. However, since our rank continuity and identifiability measures are both based on set overlap only, in both the preserved and the re-ranked case the metrics would achieve a perfect score of 1 . To take this difference into account we introduce a measure for rank instability.

\section{Rank instability}

A different way of assessing how well top-ranking nodes (or nodes in general) preserve their ranks across different thresholds is to calculate the ranges of ranks they attain. A robust metric should result in relatively narrow rank ranges. In particular, overall top ranking nodes should have relatively narrow rank ranges.

In order to quantify this behaviour we define rank instability as the scaled average rank range of the overall top 1\% ranking nodes (details in Methods). Unlike the rank continuity and identifiability measures, where values close to one represent robustness, instability values close to zero correspond to narrower rank ranges, and therefore more consistent node metric behaviour. The instability measure was lower in the HPRED network, where the mediumhigh confidence interval is shorter, and similar across the three STRING PINs (Fig. 4a and Additional file 1: Table S7). Only four metrics had instability measures below 0.01 in all PINs-number of edges in the step-1 ego network, LOUD natural connectivity, LOUD average redundancy and LOUD average number of edges in the step-1 ego network.

Rank instability measures in the synthetic networks were generally higher than in the scored PINs (Fig. 4b and Additional file 1: Table S7). Spearman correlations of metric rank instability were higher across the scored PINs than they were between PINs and either of the synthetic networks (Additional file 1: Table S4), suggesting once again that PINs exhibit context-specific behaviour.

Overall, the three measures for rank robustness of node metrics described here-rank continuity, identifiability, and instability-agree across the four studied protein interaction networks and identify four node metrics to be robust to thresholding: number of edges in the step-1 ego network, LOUD natural connectivity, LOUD average redundancy and LOUD average number of edges in the step-1 ego network. Measures of rank continuity, identifiability, and instability for all 25 analysed metrics averaged over the four PINs can be found in Additional file 1: Table S1.

\section{Discussion}

Protein interaction network analysis typically starts with network construction-some interaction data of interest 


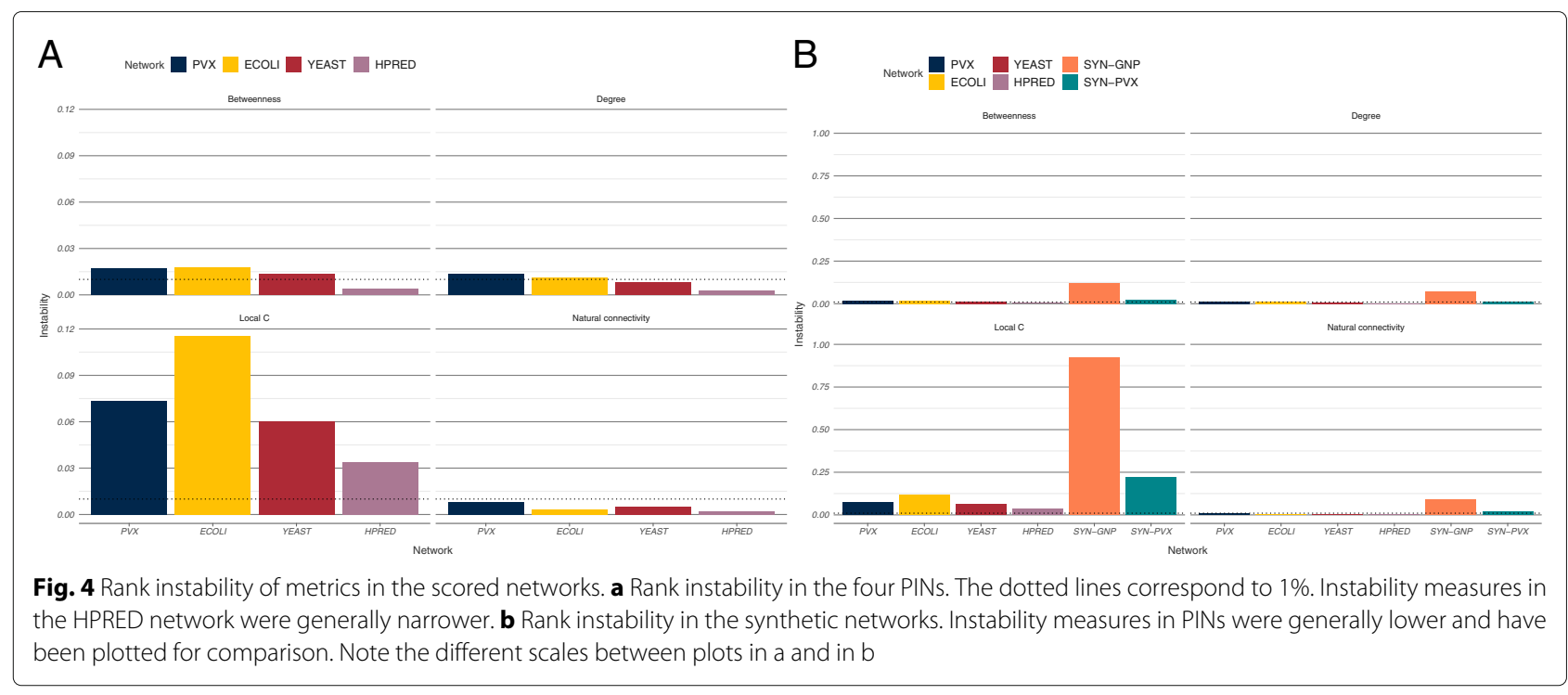

is obtained, either experimentally or from publicly available databases, and is then pre-processed before being used to create a network. Different types of protein interactions, experimental systems for detecting them, and data clean-up choices mean that multiple networks can be built to represent the same underlying biological process. Since typically only one of these will be analysed, it is not immediately clear how much these network models of cellular biology and any conclusions drawn from them might differ. In this paper we have demonstrated that varying the confidence score threshold for interactions can result in structurally different networks, both in terms of density and in terms of properties like average local clustering coefficient. We propose that if PIN analysis aims to provide reliable, reproducible biological insight, it should show some agreement across alternative network models. This property is desirable in a range of contexts: any network analysis pipeline which relies on thresholding in cases where an optimal threshold cannot be identified with high confidence should produce similar results across different thresholds. Such analysis may have different goals, such as protein function prediction or community detection, and may employ a range of tools, such as explicit node metrics and machine learning tools.

In this paper, we have focused on one frequent goal of PIN analysis-identifying key proteins (nodes) in a particular piece of biological architecture (network). This can be done by calculating node metrics, such as degree or betweenness centrality, and then identifying the set of nodes which rank highest based on metric performance. There are many different metrics one can use in this context, and it is not always clear which are the most suitable. We argue that one desirable feature a suitable metric should posses is rank robustness, or the ability to identify the same or at least largely similar sets of top ranking proteins when the network construction process is altered. Here we have considered robustness to variation in the confidence score threshold required to include an interaction in the network.

Networks constructed at lower thresholds are denser and potentially include more spurious interactions than networks constructed at higher thresholds. Increasing the stringency of data quality, however, may result in potentially important but under-studied interactions to be omitted. Different scoring procedures across databases, and even within different versions of the same database (Additional file 1: Figure S9), make optimal threshold choice difficult. Therefore, a level of rank robustness across different thresholds is desirable for node metrics. We have proposed three measures with which to assess such robustness-rank continuity, identifiability, and instability. The relevance of each measure will depend on the research question at hand and on the reliability of the confidence scoring procedure.

Rank continuity captures the effect of small threshold perturbations. This measure may be of particular interest when a narrow band of permissible thresholds has been identified. For example, if researchers are only interested in high confidence STRING interactions (threshold 0.75 ), they may wish to explore whether any metrics they use have high rank continuity scores, and in particular whether results obtained at thresholds 0.74 to 0.76 are similar.

Rank identifiability compares ranks at given thresholds against overall ranks. It may be particularly informative when there are no known optimal threshold values. For example, if researchers are uncertain of their threshold choice, they may wish to use metrics with high rank identifiability, since these metrics show good agreement across a wider range of thresholds. 
Finally, rank instability assesses the variation in threshold ranks for the overall top $1 \%$ of nodes. It is our most stringent measure, requiring not only that highly ranked proteins remain highly ranked but also that they retain their ordering across thresholds. It should be used when absolute rank is important. For example, if the researchers are not only interested in what the key (i.e. top ranking) nodes in the network are, but also how they are ordered, they should focus on metrics with low rank instability. If the top three nodes by degree were $\mathrm{A}, \mathrm{B}$ and $\mathrm{C}$ at every threshold, but these were differently ordered-say A-B-C at threshold 0.75 and C-B-A at threshold 0.76, etc.- - then rank continuity and rank identifiability would not penalise the reordering, while rank instability would.

We calculated the rank continuity, identifiability, and instability of 25 node metrics in each of six scored networks, four of which were PINs and two of which were synthetically generated. We limited ourselves to networks with fewer than 7000 nodes because of the computational cost associated with metric extraction, and in particular with calculating LOUD natural connectivity. Calculating natural connectivity for a single node at a single threshold for our largest network, the STRING network for yeast, takes approximately 88 seconds on a standard desktop computer. Since often subnetworks from higher eukaryotes, such as disease networks, are analysed, we believe our methodology will be useful for a wide range of applications.

Our rank continuity measure, which is based on a rank similarity measure originally proposed by [42], quantifies the agreement between node rankings obtained at consecutive thresholds. If networks obtained at two close thresholds, say 0.50 and 0.51 , yield considerably different rankings, this may suggest that threshold choice plays an overwhelmingly important role in network construction, and may obscure any underlying biological signal that could otherwise be detected. Conversely, high continuity measures correspond to rankings which are unlikely to significantly change with small threshold perturbations.

Confidence scores are not always readily interpretable, which might make threshold choice more difficult. Therefore, agreement over a wider threshold region might also be desirable. Small differences in consecutive thresholds might be responsible for large discrepancies between more distant thresholds (say 0.50 and 0.70), while still preserving a high rank continuity measure. In order to take this effect into account, we introduce rank identifiability to measure the agreement between different threshold rankings and a single overall ranking.

Finally, our rank instability measure provides an alternative way of analysing rank robustness which is not based on rank overlap but instead focuses on the different ranks a particular node attains at different thresholds. High instability corresponds to the overall top ranking nodes attaining a wide range of individual threshold ranks.

Our analysis identified four node metrics-number of edges in the step-one ego network, LOUD average redundancy, LOUD average number of edges in the step-one ego network, and LOUD natural connectivity-which induce robust ranks across all four analysed PINs. More commonly used metrics such as degree, local clustering coefficient, betweenness, and closeness did not perform as well. For example, when comparing the top 100 nodes obtained at the start and the end of the medium-high confidence region for the YEAST network (thresholds set at 0.60 and 0.90 respectively), node sets obtained using LOUD natural connectivity showed a three quarter overlap ( 75 out of 100). In contrast, the overlap of the top ranking sets identified by betweenness was less than half ( 41 out of 100), and the overlap between sets identified using local clustering coefficient was less than $10 \%$ (9 out of 100).

Spearman rank correlations between robustness measures across the four different PINs were consistently high ( 0.81 and above). In particular, the two yeast networksYEAST, obtained from STRING, and HPRED, obtained from HitPredict-were in good agreement, meaning that the same metrics appeared as robust across both networks, despite the different types of data and scoring procedures used (Spearman correlation coefficients for metric robustness across the two networks are 0.89 for rank continuity, 0.81 for rank identifiability, 0.92 for rank instability, Additional file 1: Tables S2-S4). The analysed PINs varied in organism, database, confidence scoring methodology, and even interaction type, yet the rank robustness results across them were very similar. This implies that the presented robustness results may be readily applicable to other PINs. In contrast, the lower correlations observed when scored PINs were compared with the two synthetic networks (Spearman correlations below 0.64, Additional file 1: Tables S2-S4) indicate that rank robustness is context-specific. The differences observed in the SYN-GNP network imply that network topology (i.e. how the edges are placed across nodes) plays a role in metric robustness. Meanwhile, the differences observer in the SYN-PVX network show that even if network topology resembles that of a PIN, how scores are allocated to network edges also plays a role in rank robustness.

\section{Conclusions}

Protein interaction data can be obtained using a range of techniques and is subject to different types of experimental error. The uncertainty associated with interaction detection can be quantified by confidence scores. Applying a threshold to these scores provides researchers with a degree of control over the false positives and false negatives present in protein interaction networks. Nevertheless, we argue that if PIN analysis aims to capture 
biological insight, it should show a level of agreement across networks obtained at different thresholds.

We present a methodology for assessing metric rank robustness in scored protein interaction networks. By analysing a set of networks from different organisms and databases, we demonstrate that metric robustness is similar across different PINs. Finally, we identify a set of node metrics which are consistently robust across different networks-therefore making our results directly applicable to other, possibly larger networks.

\section{Methods}

\section{Protein interaction and synthetic networks}

In order to assess the rank robustness of different network metrics, four scored protein interaction networks were used. The networks ranged across two databases and three organisms. A confidence score quantifying the reliability of available interaction evidence was available for each detected edge across all four networks.
Three organism networks-P. vivax (retrieved March 2018), E. coli, and S. cerevisiae (both retrieved Feb 2018) were obtained from STRING [28]. STRING contains both physical and functional association data, collected across a range of experimental and in silico interaction detection techniques. The organisms were chosen because they are model organisms with higher-than-average coverage of protein-protein interaction screens, while also having relatively small proteomes, thus reducing the computational cost of our analysis.

In order to allow for a comparison between databases, the interaction network for S. cerevisiae was also downloaded from HitPredict [29]. Unlike STRING, HitPredict is a curated database containing only high-confidence physical interactions.

Filters were applied to remove duplicate interaction records, self-interactions, and interactions to proteins of other organisms. Only combined interaction scores were considered in all four cases, ignoring any available subscores. Confidence score distributions for the four networks can be seen in Fig. 5 .

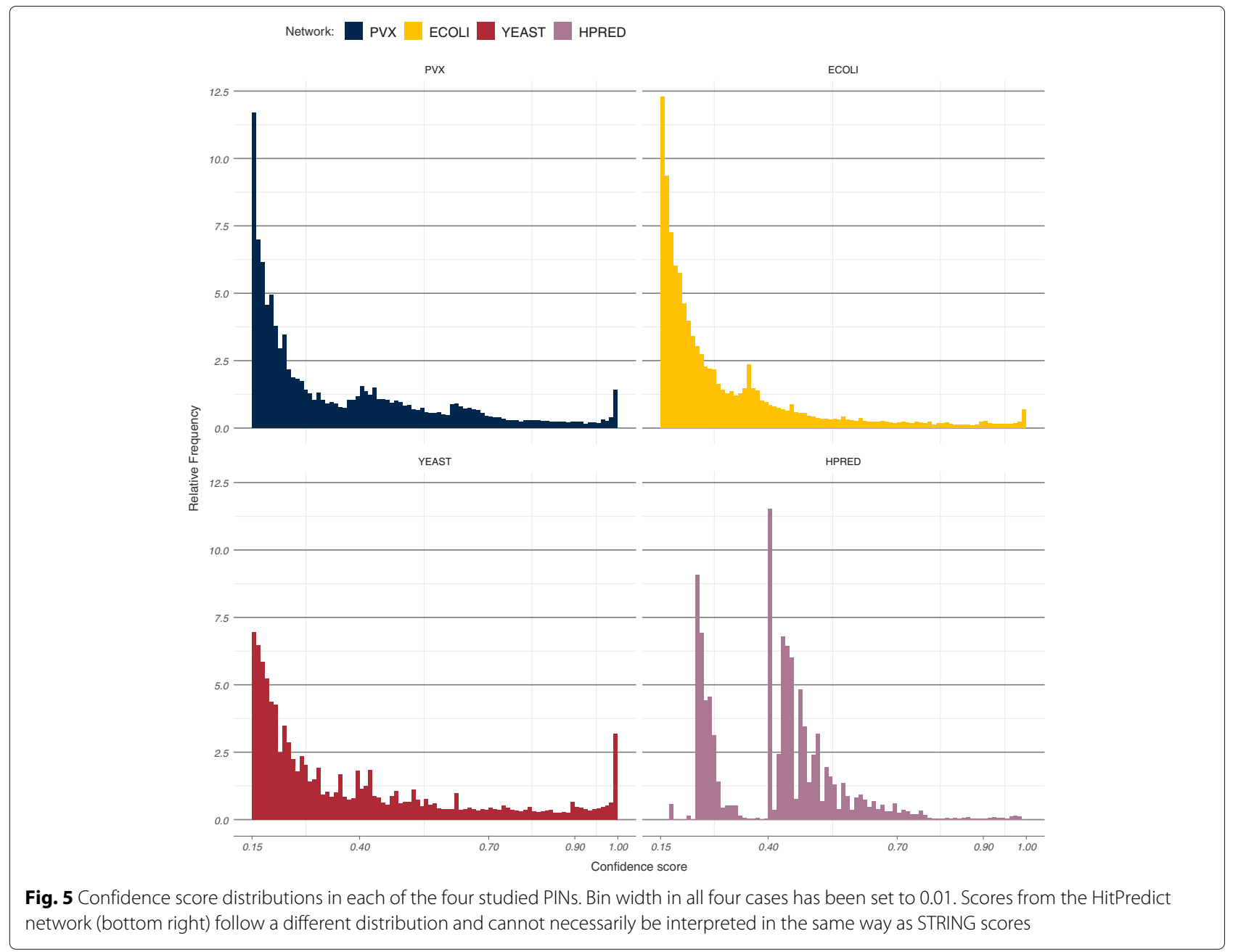


Two synthetic scored networks were also analysed. One was generated using a Bernoulli random graph model on $N=500$ nodes and with edges sampled independently at random with probability $p=0.06$. The value of $p$ was chosen to be close to network density in the STRING PINs before thresholding. Edge scores were sampled with replacement from the $P$. vivax confidence score distribution. The second network was an induced random subgraph of the $P$. vivax network on $N=1000$ nodes. The available edge scores for the subgraph were randomly rearranged and placed over the fixed edges. This resulted in a network which is PIN-like in topology, but which contains no local dependency between edge scores. A summary of all six analysed networks can be found in Table 1.

\section{Thresholding}

Applying a threshold $\theta$ to a scored PIN means discarding all edges in the network with scores strictly lower than $\theta$ and creating a simple, unweighted network from the remaining edges. The node set is not altered. Figure 6 gives a schematic of how thresholds are applied.

All reported confidence scores in the analysed PINs were between 0.15 and 1.00. Thresholds were applied from 0.15 to 0.99 inclusive at 0.01 intervals, resulting in a set of 85 distinct thresholded networks for each of the scored PINs. The same node set was preserved across all 85 thresholded networks, even when thresholding resulted in isolating nodes from the rest of the network. In order to minimise the effect of extreme thresholding, both the full threshold region and a truncated, medium-high confidence region were analysed for each network.

The majority of interactions in STRING are re-scored across different database releases (Additional file 1: Figure S9) which indicates that the scores themselves should be treated with some error tolerance. In order to take this into account while retaining score interpretability, a wide medium-high confidence region was set between 0.60 and 0.90. Medium-high confidence scores in STRING occur at similar, low frequencies across organisms (Fig. 5). HitPredict generally contains higher quality data and employs a different, more stringent scoring procedure-while interaction scoring 0.40 in STRING would be considered medium confidence, the same score in HitPredict would indicate high confidence. The truncated medium-high confidence region for the HPRED network was therefore set between 0.15 and 0.28 , since HitPredict scores above 0.28 are considered to be high-confidence [29].

An overly stringent threshold may remove so many interactions that the network structure is destroyed. However, even at the highest thresholds we consider, a giant connected component accounts for most of the nodes in each protein interaction network (91\% of nodes in PVX, $79 \%$ in ECOLI, 93\% in YEAST, and all but two nodes in HPRED).

\section{Metric extraction and ranking}

The rank robustness of twenty-five node metrics was studied. These included twelve node centralities, and thirteen leave-one-out difference (LOUD) global network summaries.

Commonly used metrics, such as degree, local clustering coefficient, betweenness and closeness centralities were included in the node metric set. In addition, metrics based on the size and density of the step-one and step-two ego networks for each node were calculated. The step-one ego network for a node $v$ was formed by taking the subgraph induced by $v$ and its immediate neighbours (graph distance 1); in addition, the step-two ego-network also included nodes within graph distance 2 from $v$.

The LOUD metrics were used as a way to assess the effect of perturbing the network by isolating each node in turn. These included, wherever applicable, local metrics averaged over the entire network (e.g. average local clustering coefficient), as well as metrics which are by definition global (e.g. global clustering coefficient). The set also included natural connectivity, a spectral metric designed to measure the overall robustness of a network [44]. Due to the associated computational costs, LOUD metrics were only calculated for nodes with degree at least two. Metric values for leaves and isolated nodes were set to NA (not available).

Table 1 Summary statistics for the six analysed networks

\begin{tabular}{lllll}
\hline Name & Network & Number of nodes & Number of edges & Edge density \\
\hline PVX & P. vivax, STRING & 3255 & 344691 & $\sim 0.065$ \\
ECOLI & E. coli, STRING & 4144 & 583440 & $\sim 0.068$ \\
YEAST & S. cerevisiae, STRING & 6418 & 939998 & $\sim 0.046$ \\
HPRED & S. cerevisiae, HitPredict & 5673 & 113001 & $\sim 0.007$ \\
SYN-GNP & Synthetic, Bernoulli & 500 & 7459 & $\sim 0.060$ \\
SYN-PVX & Synthetic, randomised P. vivax & 1000 & 30516 & $\sim 0.061$ \\
\hline
\end{tabular}

The left-most column corresponds to the names the networks are referred as later in the text. The number of edges and edge density refer to the all scored edges before any threshold is applied to the network 

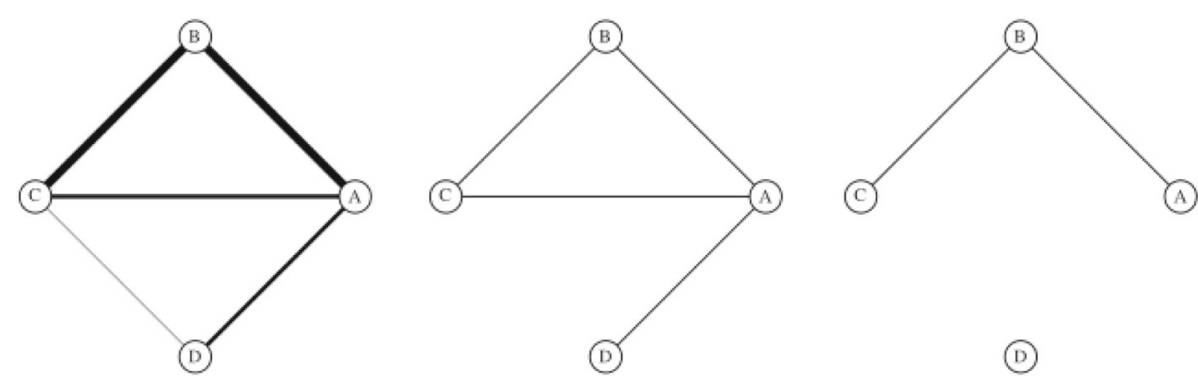

Fig. 6 Thresholding scored networks. A scored network, with edge widths corresponding to confidence scores (left). At a low threshold, only the lowest scoring edge $C D$ is removed (middle). At a higher threshold, only the highest scoring edges $A B$ and $B C$ remain in the network (right). Edge scores are otherwise ignored in the thresholded networks

The nodes in each thresholded network were ranked by each of the metrics, with high ranks corresponding to high metric values. Node ranks for nodes for which LOUD metrics were not evaluated were set to first, i.e. smallest. Ties were resolved at random, independently across different metrics and different thresholds. A full list of node metrics can be found in Table 2. Further details on their computation are given in the SI.

\section{Evaluation of rank robustness}

As the threshold applied to the scored networks increases, the networks become lower in edge density and node metric values will be affected-for example, node degrees will decrease. In order to assess the robustness of each metric to changes in threshold, the node rankings induced by the metric at different thresholds were compared instead of the calculated values.

Rank similarity is typically measured by a rank correlation coefficient such as Spearman or Kendall. These coefficients are used to compare whole rank vectors. In the context of bioinformatics applications, node metrics are often used to identify the key actors in a particular process, and therefore it is natural to focus on the highest ranking nodes only. In order to do this, robustness analysis was based on the rank similarity measure $M_{k}$ proposed by [42] as follows.

A ranking $A$ is a vector $A=\{A(v): v \in\{1, \ldots, N\}\}$ of ranks assigned to the network nodes $v \in\{1, \ldots, N\}$, e.g. by considering their degree at a particular threshold. The $k$-similarity of two rankings $A_{\theta}$ and $A_{\mu}$, obtained at thresholds $\theta$ and $\mu$ respectively, is the overlap between their $100 k \%$ highest ranking nodes, where $k \in(0 ; 1]$,

$M_{k}\left(A_{\theta}, A_{\mu} \mid k\right)=\frac{\left|\left\{v: A_{\theta}(v)>N(1-k) \wedge A_{\mu}(v)>N(1-k)\right\}\right|}{N k}$.

This measure of rank similarity is symmetric, and is therefore useful for cases where both rankings carry the same meaning, e.g. when they are obtained at consecutive thresholds. However, it is too restrictive when the rankings being compared are interpreted differently. The $\alpha$-relaxed $k$-similarity of a ranking $A$ to some ranking $B$ is the proportion of the top $100 \mathrm{k} \%$ highest ranking nodes in $B$ which are also within the set of $100 k \alpha \%$ highest ranking nodes in $A, \alpha>0$, and $k, k \alpha \in(0 ; 1]$ :

$M_{k}^{\alpha}(A, B \mid k, \alpha)=\frac{|\{v: A(v)>N(1-k \alpha) \wedge B(v)>N(1-k)\}|}{N k}$.

Our relaxed $k$-similarity allows for more user control when the rankings compared are not interpreted in the same way, and need therefore not be treated in the same way. For example, if $A$ is obtained from a single threshold $A=A_{\theta}$, and $B$ is some overall ranking, relaxed $k$-similarity may be used to identify whether the top 10 nodes overall, i.e. in $B$, are among the top 20 for the particular observed threshold, i.e. in $A_{\theta}$.

\section{Rank continuity}

We introduce rank continuity of each metric in each network, i.e. the similarity between rankings induced at consecutive thresholds. In all cases a set of values for the proportion of nodes $\mathrm{k}$ considered to be the top ranking were used, ranging from 0.001 to 0.05 at 0.001 intervals. An overall continuity measure was calculated based on how often the observed similarity was high ( 0.90 or above).

Suppose that a metric $f$ induces node rankings $A_{\mu}, A_{\mu+0.01}, \ldots, A_{\nu}$ at each threshold within the mediumhigh confidence region $[\mu, \nu]$. Then we define the rank continuity of $f$ as the fraction of cases where the $k$ similarity between consecutive $A_{\theta}$ and $A_{\theta+0.01}$ is over 0.90 for $\theta \in\{\mu, \mu+0.01, \ldots, v\}$ and $k \in[0.001,0.002, \ldots, 0.05]$ :

$$
\operatorname{rank} \text { continuity }(f) \propto\left|(\theta, k): M_{k}\left(A_{\theta}, A_{\theta+0.01} \mid k\right) \geq 0.90\right| .
$$

Since a range of different values of $k$ up to 0.05 was considered in calculating a single continuity measure, higher ranking nodes contribute more to the overall rank continuity of a metric. 
Table 2 The complete set of twenty-five standard and LOUD metrics, calculated at each node $v$

\begin{tabular}{|c|c|}
\hline Name & Details \\
\hline Degree & Number of neighbours of $v$ \\
\hline Local clustering & Proportion of pairs of neighbours of $v$ which are also connected \\
\hline Redundancy & $($ Local clustering $) \times($ Degree -1$)[43]$ \\
\hline PageRank & Calculated with the default damping factor $d=0.85[45]$ \\
\hline Closeness & Reciprocal to the sum over all $u$ of node-to-node distances $d(u, v)[46]$ \\
\hline Harmonic centrality & The sum over all $u$ of $1 / d(u, v)[47]$ \\
\hline Betweenness & Measures how many shortest paths a node $v$ contributes to [46] \\
\hline$e_{\text {one }}(v)$ & Number of edges in the step-one ego-network of $v$ \\
\hline$n_{t w o}(v)$ & Number of nodes in the step-two ego-network of $v$ \\
\hline$n_{\text {diff }}(v)$ & Number of nodes that have exactly distance two to $\mathrm{v}$ \\
\hline$n_{\text {sqdiff }}(v)$ & A measure of relative local density calculated as $n_{t w o}(v)-\operatorname{degree}(v)^{2}$ \\
\hline$n_{\text {ratio }}(v)$ & The ratio of step-one to step-two neighbourhood sizes for $v$ \\
\hline LOUD Average local clustering & $f(G)$ is the average local clustering \\
\hline LOUD Global clustering & $f(G)$ is the global clustering, i.e. the proportion of connected triplets of nodes which form triangle \\
\hline LOUD Average redundancy & $f(G)$ is the average redundancy \\
\hline LOUD Average closeness & $f(G)$ is the average closeness \\
\hline LOUD Average path length & $f(G)$ is the average path length \\
\hline LOUD Number of connected pairs & $f(G)$ is the number of pairs of nodes, which are in the same connected component \\
\hline LOUD Average betweenness & $f(G)$ is the average betweenness \\
\hline LOUD Natural connectivity & $f(G)$ is the natural connectivity [44] \\
\hline LOUD Average $e_{\text {one }}(v)$ & $f(G)$ is the average $e_{\text {one }}(v)$ \\
\hline LOUD Average $n_{t w o}(v)$ & $f(G)$ is the average $n_{\text {two }}(v)$ \\
\hline LOUD Average $n_{\text {diff }}(v)$ & $f(G)$ is the average $n_{\text {diff }}(v)$ \\
\hline LOUD Average $n_{\text {sqdiff }}(v)$ & $f(G)$ is the averagen sqdiff $(v)$ \\
\hline LOUD Average $n_{\text {ratio }}(v)$ & $f(G)$ is the average $n_{\text {ratio }}(v)$ \\
\hline
\end{tabular}

Standard metrics are above the line break. LOUD metrics are below the line break. LOUD metrics are based on global metrics $f$ calculated both for each thresholded network $G$, and for the same network, where in turn each node $v$ has been isolated from its neighbours $G_{V}$. The difference between the two metrics is recorded as $f_{L O u D}(v)=f(G)-f\left(G_{V}\right)$

\section{Rank identifiability}

Further, for each metric an overall ranking was calculated for the truncated threshold region. The overall ranks for all nodes were calculated by first averaging over node ranks at all relevant thresholds, and then ranking the resulting values. Ties were resolved at random.

For our definition of rank identifiability, for each metric the $\alpha$-relaxed $k$-similarity between overall ranks $B$ and threshold ranks $A_{\theta}$ for each threshold $\theta$ in the mediumhigh confidence region was calculated. The rank identifiability score for each metric $f$ was defined as the minimum observed relaxed similarity between overall and threshold ranks.

rankidentifiability $(f)=\min _{\theta}\left\{M_{k}^{\alpha}\left(A_{\theta}, B \mid k=100 / N, \alpha=1.5\right)\right\}$.

In all cases apart from the Bernoulli network, which had the smallest number of nodes, the ability to recover the top $n=100$ nodes overall (i.e. $k=100 / N$ ) among the top 150 (i.e. $\alpha=1.5$ ) at any threshold was tested. In the Bernoulli network, $n$ was lowered to $n=20$, and the parameter $\alpha$ remained fixed at $\alpha=1.5$.

\section{Rank instability}

Another way to assess rank robustness is through the instability of node ranks attained by different thresholds.

For each metric $f$, the top $1 \%$ overall top ranking nodes $U=\{v \mid B(v)>99 \% N\}$ were identified. Then the rank ranges attained by each of these nodes over the mediumhigh confidence region were calculated as:

$$
\operatorname{range}(v)=\max _{\theta}\left(A_{\theta}(v)\right)-\min _{\theta}\left(A_{\theta}(v)\right),
$$

where $\left\{A_{\theta}\right\}_{\theta \in[\mu, \nu]}$ are the ranks obtained from the same metric $f$ over the medium-high confidence region. For example, ubiquitin, which is always the highest degree node in the YEAST network, would have a rank range of zero. We define the rank instability as the average scaled rank range for the nodes in $U$ : 


$$
\text { rankinstability }(f)=\frac{1}{|U|} \sum_{v \in U} \text { range }(v) / N .
$$

If ranks remain very stable under $f$, then rank ranges would be low and rank instability would be close to zero. Conversely, if the ranks were relatively unstable, or nearrandom, rank ranges would be high, and rank instability would be closer to one.

Choices for all parameters discussed above were made so that rank robustness measures capture information about the highest ranking nodes. Overly stringent parameter choices would make the different measures reward only perfect or near-perfect rank agreement and ignore persistent trends of good rank overlap. Conversely, lenient parameters would reward bad as well as good rank agreement. Provided either extreme is avoided, parameters can be set in a number of ways. In test cases, we find perturbations from the values used above do not lead to qualitatively different results. While we provide these as recommended values, our methodology is fully flexible and allows users to explore different options. A worked example of metric extraction, ranking, and robustness analysis can be found in the SI.

\section{Additional file}

Additional file 1: Supplementary Information. (PDF 356 kb)

\section{Abbreviations}

ECOLI: the E. coli network obtained from STRING; HPRED: the S. cerevisiae network obtained from HitPredict; LOUD: leave-one-out difference; PIN: protein interaction network; PVX: the P. vivax network obtained from STRING; SYN-GNP: the synthetic network based on a Bernoulli random graph; SYN-PVX: the synthetic network based on P. vivax interaction data; YEAST: the S. cerevisiae network obtained from STRING

\section{Acknowledgements}

We thank Javier Pardo Díaz for critical reading of an earlier draft of this manuscript.

\section{Authors' contributions \\ LVB, GR and CMD designed the study. LVB developed the rank robustness measures, carried out data analysis, and wrote the manuscript. GR and CMD supervised the work and edited the manuscript. AWW and JW contributed to node metric choice and data acquisition, and critically reviewed the manuscript. All authors reviewed and approved the final version of the manuscript.}

\section{Funding}

This work was supported by funding from the Engineering and Physical Sciences Research Council (EPSRC) and the Medical Research Council (MRC) [grant number EP/L016044/1] and e-Therapeutics plc.

EPSRC and MRC did not play any roles in the design of the study, collection, analyses and interpretation of the data, and in writing the manuscript. AWW and JW are employed by e-Therapeutics plc; the company played no other roles in the work presented here.

\section{Availability of data and materials}

The datasets generated and analysed during the current study and accompanying software are available on the Oxford Protein Informatics Group website http://opig.stats.ox.ac.uk/resources. All R scripts use standard libraries and are compatible with R v.3.5.0 and above.
Ethics approval and consent to participate

Not applicable.

\section{Consent for publication}

Not applicable.

\section{Competing interests}

AWW and JW are employed by e-Therapeutics plc. They contributed to data acquisition and node metric choice, and critically reviewed the manuscript.

The company did not play any other role in the work presented here.

\section{Author details}

${ }^{1}$ Department of Statistics, University of Oxford, 24-29 St Giles', OX1 3LB Oxford UK. ${ }^{2}$ e-Therapeutics Plc, 17 Fenlock Rd, OX29 8LN Long Hanborough, UK.

Received: 22 January 2019 Accepted: 19 August 2019

Published online: 28 August 2019

\section{References}

1. Barabasi A-L, Oltvai ZN. Network biology: understanding the cell's functional organization. Nat Rev Genet. 2004;5(2):101.

2. Vidal M. A unifying view of 21 st century systems biology. FEBS Lett. 2009:583(24):3891-4.

3. Vidal M, Cusick ME, Barabási A-L. Interactome networks and human disease. Cell. 2011;144(6):986-98.

4. Young MP, Zimmer S, Whitmore AV. Drug molecules and biology: Network and systems aspects. Designing multi-target drugs. Cambridge: Royal Society of Chemistry; 2012, pp. 32-49.

5. Csermely P, Korcsmáros T, Kiss HJ, London G, Nussinov R. Structure and dynamics of molecular networks: a novel paradigm of drug discovery: a comprehensive review. Pharmacol Ther. 2013;138(3):333-408.

6. Creixell P, Reimand J, Haider S, Wu G, Shibata T, Vazquez M, Mustonen V, Gonzalez-Perez A, Pearson J, Sander C, et al. Pathway and network analysis of cancer genomes. Nat Methods. 2015;12(7):615.

7. Hishigaki H, Nakai K, Ono T, Tanigami A, Takagi T. Assessment of prediction accuracy of protein function from protein-protein interaction data. Yeast. 2001;18(6):523-31.

8. Sharan R, Ulitsky I, Shamir R. Network-based prediction of protein function. Mol Syst Biol. 2007;3(1):88

9. Warde-Farley D, Donaldson SL, Comes O, Zuberi K, Badrawi R, Chao P, Franz M, Grouios C, Kazi F, Lopes CT, et al. The GeneMANIA prediction server: biological network integration for gene prioritization and predicting gene function. Nucleic Acids Res. 2010;38(suppl_2):214-20.

10. Wu Q, Ye Y, Ng MK, Ho S-S, Shi R. Collective prediction of protein functions from protein-protein interaction networks. BMC Bioinformatics. 2014;15(Suppl 2):S9. BioMed Central.

11. Ideker T, Sharan R. Protein networks in disease. Genome Res. 2008;18(4): 644-52.

12. Hase T, Tanaka H, Suzuki Y, Nakagawa S, Kitano H. Structure of protein interaction networks and their implications on drug design. PLoS Comput Biol. 2009;5(10):1000550.

13. Taylor IW, Wrana JL. Protein interaction networks in medicine and disease. Proteomics. 2012;12(10):1706-16.

14. Hopkins AL. Network pharmacology: the next paradigm in drug discovery. Nat Chem Biol. 2008:4(11):682.

15. Navlakha S, Kingsford C. The power of protein interaction networks for associating genes with diseases. Bioinformatics. 2010;26(8):1057-63.

16. Abraham SA, Hopcroft LE, Carrick E, Drotar ME, Dunn K, Williamson AJ, Korfi K, Baquero P, Park LE, Scott MT, et al. Dual targeting of p53 and c-MYC selectively eliminates leukaemic stem cells. Nature. 2016:534(7607):341

17. Han L, Li K, Jin C, Wang J, Li Q, Zhang Q, Cheng Q, Yang J, Bo X, Wang S. Human enterovirus 71 protein interaction network prompts antiviral drug repositioning. Sci Rep. 2017;7:43143.

18. Berggård T, Linse $S$, James P. Methods for the detection and analysis of protein-protein interactions. Proteomics. 2007;7(16):2833-42.

19. De Las Rivas J, Fontanillo C. Protein-protein interactions essentials: key concepts to building and analyzing interactome networks. PLoS Comput Biol. 2010;6(6):1000807

20. Lehne B, Schlitt T. Protein-protein interaction databases: keeping up with growing interactomes. Hum Genomics. 2009;3(3):291. 
21. Basha O, Flom D, Barshir R, Smoly I, Tirman S, Yeger-Lotem E. MyProteinNet: build up-to-date protein interaction networks for organisms, tissues and user-defined contexts. Nucleic Acids Res. 2015;43(W1):258-63.

22. Fields S, Song O-k. A novel genetic system to detect protein protein interactions. Nature. 1989;340(6230):245-6.

23. Rigaut G, Shevchenko A, Rutz B, Wilm M, Mann M, Séraphin B. A generic protein purification method for protein complex characterization and proteome exploration. Nat Biotechnol. 1999;17(10):1030-2.

24. D'haeseleer P, Liang S, Somogyi R. Genetic network inference: from co-expression clustering to reverse engineering. Bioinformatics. 2000;16(8):707-26.

25. Deane CM, Salwiński $Ł$, Xenarios I, Eisenberg D. Protein interactions two methods for assessment of the reliability of high throughput observations. Mol Cell Proteomics. 2002;1(5):349-56.

26. Huang $\mathrm{H}$, Bader JS. Precision and recall estimates for two-hybrid screens. Bioinformatics. 2009;25(3):372-8.

27. Dunham WH, Mullin M, Gingras A-C. Affinity-purification coupled to mass spectrometry: Basic principles and strategies. Proteomics. 2012;12(10):1576-90.

28. Szklarczyk D, Franceschini A, Wyder S, Forslund K, Heller D, Huerta-Cepas J, Simonovic M, Roth A, Santos A, Tsafou KP, et al. STRING v10: protein-protein interaction networks, integrated over the tree of life. Nucleic Acids Res. 2014;43(D1):447-52.

29. López Y, Nakai K, Patil A. HitPredict version 4: comprehensive reliability scoring of physical protein-protein interactions from more than 100 species. Database: J Biol Databases Curation. 2015;2015:bav117.

30. Orchard S, Ammari M, Aranda B, Breuza L, Briganti L, Broackes-Carter $F$, Campbell NH, Chavali G, Chen C, Del-Toro N, et al. The MlntAct project-IntAct as a common curation platform for 11 molecular interaction databases. Nucleic Acids Res. 2013;42:1115.

31. Alanis-Lobato G, Andrade-Navarro MA, Schaefer MH. HIPPIE v2. O: enhancing meaningfulness and reliability of protein-protein interaction networks. Nucleic Acids Res. 2016:45:985.

32. Krogan NJ, Cagney G, Yu H, Zhong G, Guo X, Ignatchenko A, Li J, Pu S, Datta N, Tikuisis AP, et al. Global landscape of protein complexes in the yeast Saccharomyces cerevisiae. Nature. 2006;440(7084):637.

33. Hart GT, Ramani AK, Marcotte EM. How complete are current yeast and human protein-interaction networks? Genome Biol. 2006;7(11):120.

34. Rolland T, Taşan M, Charloteaux B, Pevzner SJ, Zhong Q, Sahni N, Yi S, Lemmens I, Fontanillo C, Mosca R, et al. A proteome-scale map of the human interactome network. Cell. 2014;159(5):1212-26.

35. Newman M. Network structure from rich but noisy data. Nat Phys. 2018;14(6):542.

36. Wang DJ, Shi X, McFarland DA, Leskovec J. Measurement error in network data: A re-classification. Soc Networks. 2012;34(4):396-409.

37. Hu Y, Vinayagam A, Nand A, Comjean A, Chung V, Hao T, Mohr SE, Perrimon N. Molecular interaction search tool (MIST): an integrated resource for mining gene and protein interaction data. Nucleic Acids Res. 2017:46(D1):567-74

38. Asur S, Ucar D, Parthasarathy S. An ensemble framework for clustering protein-protein interaction networks. Bioinformatics. 2007;23(13):29-40.

39. Martin T, Ball B, Newman ME. Structural inference for uncertain networks. Phys Rev E. 2016;93(1):012306.

40. Alvarez-Ponce D. Recording negative results of protein-protein interaction assays: an easy way to deal with the biases and errors of interactomic data sets. Brief Bioinform. 2016;18(6):1017-20.

41. Curto C. What can topology tell us about the neural code?. Bull Am Math Soc. 2017:54(1):63-78

42. Trajanovski S, Martín-Hernández J, Winterbach W, Van Mieghem P. Robustness envelopes of networks. J Compl Netw. 2013;1(1):44-62.

43. Borgatti SP. Structural holes: Unpacking Burt's redundancy measures. Connections. 1997;20(1):35-8.

44. Jun W, Barahona M, Yue-Jin T, Hong-Zhong D. Natural connectivity of complex networks. Chin Phys Lett. 2010;27(7):078902.

45. Brin S, Page $L$. The anatomy of a large-scale hypertextual web search engine. Comput Netw ISDN Syst. 1998:30(1-7):107-17.

46. Freeman LC. Centrality in social networks conceptual clarification. Soc Networks. 1978;1(3):215-39.

47. Marchiori M, Latora V. Harmony in the small-world. Physica A: Stat Mech Appl. 2000;285(3-4):539-46.

\section{Publisher's Note}

Springer Nature remains neutral with regard to jurisdictional claims in published maps and institutional affiliations.
Ready to submit your research? Choose BMC and benefit from:

- fast, convenient online submission

- thorough peer review by experienced researchers in your field

- rapid publication on acceptance

- support for research data, including large and complex data types

- gold Open Access which fosters wider collaboration and increased citations

- maximum visibility for your research: over 100M website views per year

At $B M C$, research is always in progress.

Learn more biomedcentral.com/submission 